\title{
Association of alanine transaminase to aspartate aminotransferase ratio and incident diabetes in Chinese adults: a second retrospective cohort study
}

Zhilin Liang ( $\sim$ liangzhilin@email.szu.edu.cn )

Shenzhen University https://orcid.org/0000-0003-1811-9934

Haofei Hu

Shenzhen Second People's Hospital

Xunxun Wang

Shenzhen Second People's Hospital

Xianxiong Chen

Shenzhen University

Min Zhang

Shenzhen Second People's Hospital

Zhigang Liu

Shenzhen University

Research

Keywords: Alanine transaminase, Aspartate aminotransferase, ALT/AST ratio, Incident diabetes

Posted Date: May 10th, 2020

DOl: https://doi.org/10.21203/rs.3.rs-26173/v1

License: (1) This work is licensed under a Creative Commons Attribution 4.0 International License.

Read Full License 


\section{Abstract}

Aims/Introduction: Previous studies have indicated that serum alanine aminotransferase (ALT) level, serum aspartate aminotransferase (AST) level or ALT/AST ratio may be bound up with diabetes. But the association of ALT/AST ratio and incident diabetes in Chinese adults have not been elaborated yet. The main objective of the present study was to analyze whether ALT/AST ratio was an independent risk factor which attached to the risk of incident diabetes in Chinese adults.

Materials and Methods From 2010 to 2016, 685,277 Chinese adults who received a health check in Rich Healthcare Group including all medical records, and the present article was a second retrospective cohort analysis based on above population. As the target independent variable, ALT/AST ratio was measured at baseline while the dependent variable, incident diabetes appeared during follow-up. We used Cox proportional hazard regression models to analyze the prognostic value of ALT/AST on the risk of incident diabetes.

Results ALT/AST demonstrated undeniable correlation with incident diabetes ( $\mathrm{HR}=2.535,95 \% \mathrm{Cl}: 2.190$ to $2.934, \mathrm{P}<0.00001)$. The association between ALT/AST and incident diabetes is only linear. The cumulative incidence $(95 \% \mathrm{Cl})$ rate of incident diabetes in total participants, ALT/AST quartile 1, 2, 3, and 4 was 2.112 (2.016-2.208), 0.992 (.0.857-1.127), 1.430 (1.274-1.586), 2.396 (2.192-2.600) and 3.559 (3.314-3.803), separately. The population with age (30 to $<40$ years), BMI ( $<24 \mathrm{~kg} / \mathrm{m} 2)$ and SBP (< $140 \mathrm{mmHg}$ ) was found to be the stronger association in subgroup analysis.

Conclusion In Chinese adults, ALT/AST ratio is an independent risk factor for incident diabetes.

\section{Introduction}

Diabetes, a chronic disease lasting for life which not only imposes an enormous burden on patients and their families but also on society and national healthcare systems, is a huge health problem in China which has the largest diabetes epidemic in the world. More than $10 \%$ Chinese adults have diabetes according to a recent nationwide survey [1]. In 2015, 415 million people were estimated to have diabetes worldwide which is expected to reach 642 million by 2040 and more than $55 \%$ of the number occurring in Asia, quoted from the International Diabetes Federation [2]. During 2006 to 2015, WHO estimates that China have lost approximately $\$ 558$ billion of its national income to heart disease, stroke, and diabetes.

The liver which plays a significant role in the maintenance of normal glucose levels has been involved in the pathologic process of type 2 diabetes (T2DM), and hepatic dysfunction from insulin resistance syndrome has been considered as guiding factor to T2DM[3]. Pyruvate and glutamate which formed through alanine and a-ketoglutarate, play a pivotal role in glucose and protein metabolism, and alanine transaminase (ALT) is an negotiator enzyme which reversibly catalyzes transamination[4]. ALT and aspartate aminotransferase (AST) are the special marker of hepatic injury that were normally detected in diabetes[5]. Many studies have indicated that serum ALT level, serum AST level or ALT/AST ratio may be bound up with diabetes [6-9]. But these studies mostly paid attention to investigate whether ALT, AST or 
ALT/AST ratio were related to insulin resistance, the association of ALT/AST ratio and incident diabetes have not been elaborated yet, and they did not focus on the Chinese population by the way. Hence, the primary purpose of the present article was to analyze whether ALT/AST ratio was an independent risk factor which attached to the risk of incident diabetes in Chinese adults which conducted a large cohort population.

In the present article, we accomplished a secondary retrospective cohort analysis based on previously published data which the author have investigated the association between BMI and age with incident diabetes [10]. Through our analysis, the results suggested that ALT/AST ratio was used as an independent risk factor, and the result variables and covariates in accordance with those in the primal study.

\section{Methods}

\section{Data source and participants}

We acquired raw data from 'DATADRYAD' database (www.Datadryad.org), a website that allowed subscribers to download data at liberty. Abided by Dryad Terms, we quoted Dryad data package in this present study (Dryad data package: Ying Chen, Xiao-Ping Zhang, Jie Yuan, Bo Cai, Xiao-Li Wang, Xiao-Li Wu, Yue-Hua Zhang, Xiao-Yi Zhang, Tong Yin, Xiao-Hui Zhu, Yun-Juan Gu1, Shi-Wei Cui, Zhi-Qiang Lu, Xiao-Ying Li(2018) Data from: Association of body mass index and age with incident diabetes in Chinese adults: a population-based cohort study. Dryad Digital Repository. http://dx.doi.org/10.1136/bmjopen2018-021768). The database file included following variables: age, body mass index (BMI), fasting blood glucose (FPG), systolic blood pressure (SBP), diastolic blood pressure (DBP), cholesterol, triglyceride, Serum creatinine (SCR), ALT, AST, gender, high density lipoprotein cholesterol (HDL), low density lipoprotein cholesterol(LDL), family history of diabetes, smoking status, drinking status, year of follow up and censor of diabetes at follow up. The original study authors have given up all copyright and related ownership of the data. So, we can use all the data for a second analysis without transgressing the interest of authors.

Data which enrolled 685,277 participants $\geq 20$ years old who received a health check with at least two visits from 2010 to 2016, were provided by the Rich Healthcare Group in China across 32 sites and 11 cities in China(Shanghai, Beijing, Nanjing, Suzhou, Shenzhen, Changzhou, Chengdu, Guangzhou, Hefei, Wuhan, Nantong). The data has been already excluded participants by follow rules: (1) diagnosed with diabetes at baseline, (2) follow-up time less than 2 years, (3) no acquirable message about gender, height, weight or fasting plasma glucose level at baseline, (4) with immoderate BMI values $\left(<15 \mathrm{~kg} / \mathrm{m}^{2}\right.$ or $>$ $55 \mathrm{~kg} / \mathrm{m}^{2}$ ), (5) with undefined diabetes status. Ultimately, 211,833 participants were brought into the analysis by Ying Chen, et al, and the details about the inclusion/exclusion standard and result variables of the trial had been informed in the article of Ying Chen, et al[10]. On account of the character of retrospective cohort analysis, informed consent or study approval of the patient was not demanded by the institutional ethics committee. In order to research forwardly, we excluded the missing data of 
baseline ALT $(n=1782)$ and AST $(n=123290)$. Then, the ALT/AST ratio was calculated as ALT divided by AST, 87,881 subjects were elicited. We also removed the ALT/AST outliers $(n=1222)$ by the rules as follow: (1) lower than means minus three standard deviation (SD), (2) higher than means plus three SD). Ultimately, 86,659 participants including 48,942 males and 37,717 females were brought into our study for further research.

\section{Study excogitation and data measurement}

The excogitation of the study was referenced from the article of Ying Chen, et al, and the data we obtained for the present retrospective cohort analysis originated from that article too [10]. For clarifying our study process, we would list the procedure of our study as follows. Every single participant who visited the health check center, would finish a questionnaire as required. The detail of the questionnaire included demographic characteristics, personal medical history, family history of chronic disease and lifestyle factors as well. Trained staff would measure and collect the data of height which was measured to the nearest $0.1 \mathrm{~cm}$, weight which was measured in light clothing with no shoes to the nearest $0.1 \mathrm{~kg}$ and blood pressure which was measured by standard mercury sphygmomanometers of every single participant. BMI was calculated by deriving from weight in kilograms divided by height in metres squared. An autoanalyzer named Beckman 5800 was used to test FPG, cholesterol, triglyceride, SCR, ALT, AST, HDL and LDL. The target independent variable, ALT/AST was acquired at baseline and the dependent variable, the risk of incident diabetes was acquired during the following up. On account of the character of retrospective cohort analysis, we need to minify the observation bias and selection bias.

\section{Confirmation of incident diabetes}

Incident diabetes' confirmation comply with fasting plasma glucose whose over $7.00 \mathrm{mmol} / \mathrm{L}$ and/or self-reported diabetes during the follow-up. We evaluated the participants when they were diagnosed or arrived the last visit. The data of those participants who lost follow-up also consisted in the whole study.

\section{Statistical analyses}

In the first place, we filled the missing data with the median or mean value when the missing data of the variable was continuous and approached the category variable when it was categorical[11]. Secondly, we separated all participants by quartiles of ALT/AST. We used medians (quartiles, skewed distribution) or means \pm standard deviations (normal distribution) to represent the continuous variables and expressed the categorical variables by percentages or frequency. The incident diabetes rate was calculated on the whole and use the log-rank test to compare and then used cumulative incidence and person-years incidence rate to represented. In addition, we used the Kruskal-Wallis $\mathrm{H}$ (skewed distribution) test, the oneway ANOVA (normal distribution) and chi-square test (categorical variables) to pick out the evidential differences among the means and proportions in the groups. Moreover, the prognostic value of ALT/AST on the risk of incident diabetes was explored by Cox proportional hazard regression models and hazard ratios (HRs) which were adjusted by $95 \%$ confidence intervals (Cls) were figured out to assess the incident diabetes risk. Besides, we concurrently manifested the results into unadjusted, minimally adjusted and fully adjusted analyses according to the recommendation of the STROBE statement [12]. 
When the covariances was enrolled in the model and they have changed the matched hazard ratio at least by $10 \%$, we treated them as adjusted[13]. We also conducted a sensitivity analysis by regenerating the ALT/AST as a categorical variable and counting the $\mathrm{P}$ for trend that would help further confirm as a continuous variable and check the possibility of nonlinearity for the robustness of analysis. Furthermore, generalized additive models (GAM) was applied to determine the non-linear relationship and once we checked a non-linear relationship, we would count the threshold effect of the ALT/AST on incident diabetes according to the smoothing plot by conducting a two-piecewise linear regression model. If a smooth curve emerged between ALT/AST and incident diabetes, the inflection point would be counted voluntarily by using recursive method (maximum pseudo likelihood estimation model). What is more, we used Cox proportional hazard models to investigate other subgroups (age, gender, BMI, FPG, SBP, DBP, HDL, LDL, family history of diabetes, smoking status, drinking status) for making sure of the characteristic of analysis. We replaced the continuous variable categorically in terms of their clinical demarcation point and adjusted every single stratification for all the mixed factors. We also used likelihood ration tests to examine the modificatory and interaction of the subgroups. Through the Kaplan-Meier method specifically the time-to-first event for each endpoint, we canvassed the survival estimate and cumulative event rate. As for adverse event, we used the log- rank test to examine the Kaplan-Meier hazard ratios (HR) and the $95 \%$ confidence intervals (Cls) of them.

All statistical analysis mentioned above was utilized by Empower-Stats (http://www.empowerstats.com, X\&Y Solutions, Inc., Boston, MA) or the R Project (http://www.R-project.org, The R Foundation) and statistically significant was confirmed when the $P$ values less than 0.05 .

\section{Results}

In the aggregate of 86,659 participants ( $57 \%$ male), the average age of the them was $42.23 \pm 12.82$ years. 1826 participants occurred incident diabetes during the following up with the mean following-up time of $3.14 \pm 0.92$ years. The average value of ALT/AST was $0.92 \pm 0.36$, and the average value of BMI, FPG, SBP, DBP, cholesterol, triglyceride and SCR were $23.22 \pm 3.30 \mathrm{~kg} / \mathrm{m}^{2}, 4.93 \pm 0.62 \mathrm{mmol} / \mathrm{L}, 119.24 \pm$ $16.52 \mathrm{mmHg}, 74.10 \pm 10.85 \mathrm{mmHg}, 4.69 \pm 0.90 \mathrm{mmol} / \mathrm{L}, 1.32 \pm 1.01 \mathrm{mmol} / \mathrm{L}$ and $71.21 \pm 15.69 \mathrm{mmol} / \mathrm{L}$, separately. The quantity of missing data of SBP, DBP, cholesterol, triglyceride, SCR, HDL, LDL, smoking and drinking status were $12,13,1080,1080,3475,37600,36761,64967$ and 64967 , respectively.

\section{Baseline characteristics}

Quartiles of the ALT/AST and the total population's baseline characteristics were shown in Table 1. We distributed the total population into subgroup according to ALT/AST quartiles $(\leq 0.64,0.64-0.83,0.83-$ $1.10,>1.10)$. In higher ALT/AST group, participants were observed mostly have higher BMI, fasting plasma glucose, SBP, DBP, cholesterol, SCR and more current smoking and drinking status. On the contrary, none of family history status was considered statistically significant difference amongst Quartiles of the ALT/AST. 
Table 1

Baseline Characteristics of participants

\begin{tabular}{|c|c|c|c|c|c|}
\hline ALT/AST & $\mathrm{Q1}(\leq 0.64)$ & $\begin{array}{l}\text { Q2(0.64 to } \leq \\
0.83)\end{array}$ & $\begin{array}{l}\mathrm{Q} 3(0.83 \text { to } \leq \\
1.10)\end{array}$ & Q4(®1.10) & $\begin{array}{l}\mathrm{P} \text { - } \\
\text { value }\end{array}$ \\
\hline$N$ & 20673 & 22378 & 21574 & 22034 & \\
\hline Age (years) & $42.15 \pm 14.33$ & $43.09 \pm 13.47$ & $43.15 \pm 12.55$ & $40.53 \pm 10.51$ & $<.001$ \\
\hline $\mathrm{BMI}\left(\mathrm{kg} / \mathrm{m}^{2}\right)$ & $21.50 \pm 2.74$ & $22.42 \pm 2.94$ & $23.62 \pm 3.07$ & $25.24 \pm 3.20$ & $\begin{array}{l}< \\
0.001\end{array}$ \\
\hline FPG (mmol/L) & $4.85 \pm 0.60$ & $4.89 \pm 0.61$ & $4.96 \pm 0.61$ & $5.01 \pm 0.63$ & $\begin{array}{l}< \\
0.001\end{array}$ \\
\hline $\mathrm{SBP}(\mathrm{mmHg})$ & $\begin{array}{l}115.44 \pm \\
16.77\end{array}$ & $117.78 \pm 16.55$ & $120.38 \pm 16.32$ & $\begin{array}{l}123.18 \pm \\
15.42\end{array}$ & $\begin{array}{l}< \\
0.001\end{array}$ \\
\hline $\mathrm{DBP}(\mathrm{mmHg})$ & $71.38 \pm 10.38$ & $72.87 \pm 10.54$ & $74.86 \pm 10.76$ & $77.17 \pm 10.82$ & $<0.001$ \\
\hline $\begin{array}{l}\text { Cholesterol } \\
(\mathrm{mmol} / \mathrm{L})\end{array}$ & $4.58 \pm 0.88$ & $4.62 \pm 0.89$ & $4.71 \pm 0.89$ & $4.85 \pm 0.91$ & $\begin{array}{l}< \\
0.001\end{array}$ \\
\hline $\begin{array}{l}\text { Triglyceride } \\
(\mathrm{mmol} / \mathrm{L})\end{array}$ & $\begin{array}{l}0.82(0.00- \\
16.90)\end{array}$ & $\begin{array}{l}0.95(0.00- \\
18.35)\end{array}$ & $\begin{array}{l}4.65(0.44- \\
11.65)\end{array}$ & $\begin{array}{l}1.44(0.00- \\
27.20)\end{array}$ & $\begin{array}{l}< \\
0.001\end{array}$ \\
\hline SCR (umol/L) & $66.36 \pm 15.95$ & $68.95 \pm 15.33$ & $72.93 \pm 15.75$ & $76.35 \pm 13.83$ & $\begin{array}{l}<.001 \\
0.001\end{array}$ \\
\hline ALT/AST & $0.53 \pm 0.08$ & $0.74 \pm 0.05$ & $0.96 \pm 0.08$ & $1.42 \pm 0.25$ & $\begin{array}{l}< \\
0.001\end{array}$ \\
\hline Gender & & & & & $\begin{array}{l}< \\
0.001\end{array}$ \\
\hline male & $\begin{array}{l}6101 \\
(29.51 \%)\end{array}$ & $\begin{array}{l}10154 \\
(45.37 \%)\end{array}$ & $\begin{array}{l}14067 \\
(65.20 \%)\end{array}$ & $\begin{array}{l}18620 \\
(84.51 \%)\end{array}$ & \\
\hline female & $\begin{array}{l}14572 \\
(70.49 \%)\end{array}$ & $\begin{array}{l}12224 \\
(54.63 \%)\end{array}$ & 7507 (34.80\%) & $\begin{array}{l}3414 \\
(15.49 \%)\end{array}$ & \\
\hline HDL (mmol/L) & & & & & $\begin{array}{l}<.001 \\
0.001\end{array}$ \\
\hline $\operatorname{low}(0$ to $\leq 1.23)$ & $\begin{array}{l}2649 \\
(12.81 \%)\end{array}$ & 3699 (16.53\%) & $4416(20.47 \%)$ & $\begin{array}{l}5508 \\
(25.00 \%)\end{array}$ & \\
\hline $\begin{array}{l}\text { middle }(1.24 \text { to } \leq \\
1.46)\end{array}$ & $\begin{array}{l}3748 \\
(18.13 \%)\end{array}$ & $4143(18.51 \%)$ & 4094 (18.98\%) & $\begin{array}{l}3837 \\
(17.41 \%)\end{array}$ & \\
\hline
\end{tabular}

Values are $\mathrm{n}(\%)$, median(Q1-Q3) or mean $\pm \mathrm{sd}$

$A L T$ alanine aminotransferase, $A S T$ aspartate transaminase, $B M /$ body mass index, $S B P$ systolic blood pressure, $D B P$ diastole blood pressure, $F P G$ fasting plasma glucose, $L D L$ low-density lipoprotein cholesterol, HDL high density lipoprotein cholesterol, SCR serum creatinine 


\begin{tabular}{|c|c|c|c|c|c|}
\hline ALT/AST & $\mathrm{Q1}(\leq 0.64)$ & $\begin{array}{l}\text { Q2(0.64 to } \leq \\
0.83)\end{array}$ & $\begin{array}{l}\text { Q3 }(0.83 \text { to } \leq \\
1.10)\end{array}$ & Q4(囚1.10) & $\begin{array}{l}\mathrm{P}- \\
\text { value }\end{array}$ \\
\hline $\begin{array}{l}\operatorname{high}(1.47 \text { to } \leq \\
10.40)\end{array}$ & $\begin{array}{l}5179 \\
(25.05 \%)\end{array}$ & $4814(21.51 \%)$ & 3960 (18.36\%) & $\begin{array}{l}3012 \\
(13.67 \%)\end{array}$ & \\
\hline not recorded & $\begin{array}{l}9097 \\
(44.00 \%)\end{array}$ & $9722(43.44 \%)$ & 9104 (42.20\%) & $\begin{array}{l}9677 \\
(43.92 \%)\end{array}$ & \\
\hline LDL (mmol/L) & & & & & $\dot{L}_{0.001}$ \\
\hline $\operatorname{low}(0$ to $\leq 2.42)$ & $\begin{array}{l}4577 \\
(22.14 \%)\end{array}$ & $4570(20.42 \%)$ & 3891 (18.04\%) & $\begin{array}{l}3343 \\
(15.17 \%)\end{array}$ & \\
\hline $\begin{array}{l}\text { middle(2.42 to } \leq \\
2.98)\end{array}$ & $\begin{array}{l}4116 \\
(19.91 \%)\end{array}$ & 4307 (19.25\%) & $4272(19.80 \%)$ & $\begin{array}{l}4064 \\
(18.44 \%)\end{array}$ & \\
\hline $\begin{array}{l}\text { high(2.99 to } \leq \\
10.07)\end{array}$ & $\begin{array}{l}3366 \\
(16.28 \%)\end{array}$ & 4011 (17.92\%) & $4412(20.45 \%)$ & $\begin{array}{l}4969 \\
(22.55 \%)\end{array}$ & \\
\hline not recorded & $\begin{array}{l}8614 \\
(41.67 \%)\end{array}$ & $9490(42.41 \%)$ & 8999 (41.71\%) & $\begin{array}{l}9658 \\
(43.83 \%)\end{array}$ & \\
\hline $\begin{array}{l}\text { Family history of } \\
\text { diabetes }\end{array}$ & & & & & 0.028 \\
\hline no & $\begin{array}{l}20255 \\
(97.98 \%)\end{array}$ & $\begin{array}{l}21955 \\
(98.11 \%)\end{array}$ & $\begin{array}{l}21165 \\
(98.10 \%)\end{array}$ & $\begin{array}{l}21540 \\
(97.76 \%)\end{array}$ & \\
\hline yes & $418(2.02 \%)$ & $423(1.89 \%)$ & 409 (1.90\%) & 494 (2.24\%) & \\
\hline Smoking status & & & & & $\begin{array}{l}<.001 \\
0.00\end{array}$ \\
\hline current & $526(2.54 \%)$ & 795 (3.55\%) & $1101(5.10 \%)$ & $1663(7.55 \%)$ & \\
\hline ever & 117 (0.57\%) & 174 (0.78\%) & 281 (1.30\%) & 393 (1.78\%) & \\
\hline never & $\begin{array}{l}4566 \\
(22.09 \%)\end{array}$ & 4275 (19.10\%) & 3898 (18.07\%) & $\begin{array}{l}3903 \\
(17.71 \%)\end{array}$ & \\
\hline not recorded & $\begin{array}{l}15464 \\
(74.80 \%)\end{array}$ & $\begin{array}{l}17134 \\
(76.57 \%)\end{array}$ & $\begin{array}{l}16294 \\
(75.53 \%)\end{array}$ & $\begin{array}{l}16075 \\
(72.96 \%)\end{array}$ & \\
\hline Drinking status & & & & & $\begin{array}{l}< \\
0.001\end{array}$ \\
\hline current & $139(0.67 \%)$ & $132(0.59 \%)$ & $136(0.63 \%)$ & $164(0.74 \%)$ & \\
\hline ever & 547 (2.65\%) & 761 (3.40\%) & 996 (4.62\%) & $1443(6.55 \%)$ & \\
\hline
\end{tabular}

Values are $\mathrm{n}(\%)$, median(Q1-Q3) or mean $\pm \mathrm{sd}$

$A L T$ alanine aminotransferase, $A S T$ aspartate transaminase, $B M /$ body mass index, $S B P$ systolic blood pressure, $D B P$ diastole blood pressure, $F P G$ fasting plasma glucose, $L D L$ low-density lipoprotein cholesterol, HDL high density lipoprotein cholesterol, SCR serum creatinine 


\begin{tabular}{|c|c|c|c|c|c|}
\hline ALT/AST & $\mathrm{Q1}(\leq 0.64)$ & $\begin{array}{l}\text { Q2(0.64 to } \leq \\
0.83)\end{array}$ & $\begin{array}{l}\mathrm{Q} 3(0.83 \text { to } \leq \\
1.10)\end{array}$ & Q4(囚1.10) & $\begin{array}{l}\mathrm{P}- \\
\text { value }\end{array}$ \\
\hline never & $\begin{array}{l}4523 \\
(21.88 \%)\end{array}$ & $4351(19.44 \%)$ & $4148(19.23 \%)$ & $\begin{array}{l}4352 \\
(19.75 \%)\end{array}$ & \\
\hline not recorded & $\begin{array}{l}15464 \\
(74.80 \%)\end{array}$ & $\begin{array}{l}17134 \\
(76.57 \%)\end{array}$ & $\begin{array}{l}16294 \\
(75.53 \%)\end{array}$ & $\begin{array}{l}16075 \\
(72.96 \%)\end{array}$ & \\
\hline \multicolumn{6}{|c|}{ Values are $n(\%)$, median(Q1-Q3) or mean \pm sd } \\
\hline \multicolumn{6}{|c|}{$\begin{array}{l}A L T \text { alanine aminotransferase, } A S T \text { aspartate transaminase, } B M I \text { body mass index, } S B P \text { systolic } \\
\text { blood pressure, } D B P \text { diastole blood pressure, } F P G \text { fasting plasma glucose, } L D L \text { low-density lipoprotein } \\
\text { cholesterol, } H D L \text { high density lipoprotein cholesterol, } S C R \text { serum creatinine }\end{array}$} \\
\hline
\end{tabular}

\section{Incidence rate of incident diabetes}

Table 2 showed that 1,826 (2.11\%), 205 (0.99\%), 320 (1.43\%), 517 (2.40\%), 784 (3.56\%) participants developed incident diabetes during the following up in total participants, ALT/AST quartile 1, 2, 3, and 4, respectively. The incidence rate of incident diabetes was $671.79,318.73,452.34,761.29,1138.85$ per 100,000 person-years the aggregate of participants, ALT/AST quartile 1, 2, 3, and 4, respectively. And the cumulative incidence (95\% Cl) was 2.112 (2.016-2.208), 0.992 (.0.857-1.127), 1.430 (1.274-1.586), 2.396 (2.192-2.600) and 3.559 (3.314-3.803), respectively. These results indicated that individual with higher ALT/AST ratio had a higher cumulative incidence.

Table 2

Incidence rate of incident diabetes

\begin{tabular}{|c|c|c|c|c|}
\hline Group & $\begin{array}{l}\text { No of } \\
\text { participants }\end{array}$ & $\begin{array}{l}\text { No of DM } \\
\text { events }\end{array}$ & $\begin{array}{l}\text { Cumulative incidence } \\
(95 \% \mathrm{Cl})\end{array}$ & $\begin{array}{l}\text { Per } 100,000 \\
\text { person-years }\end{array}$ \\
\hline Total & 86659 & $\begin{array}{l}1826 \\
(2.11 \%)\end{array}$ & $2.112(2.016-2.208)$ & 671.79 \\
\hline \multicolumn{5}{|l|}{$\begin{array}{l}\text { ALT/AST } \\
\text { (quartile) }\end{array}$} \\
\hline Q1 & 20468 & 205 (0.99\%) & $0.992(.0 .857-1.127)$ & 318.73 \\
\hline Q2 & 22058 & $320(1.43 \%)$ & $1.430(1.274-1.586)$ & 452.34 \\
\hline Q3 & 21057 & $517(2.40 \%)$ & $2.396(2.192-2.600)$ & 761.29 \\
\hline Q4 & 21250 & $784(3.56 \%)$ & $3.559(3.314-3.803)$ & 1138.85 \\
\hline $\begin{array}{l}\text { P-value for log- } \\
\text { rank test }\end{array}$ & - & - & $\triangle 0.001$ & - \\
\hline
\end{tabular}

\section{Univariate analysis}

The consequence of univariate analyses was demonstrated in Table 3 that age, BMI, FPG, SBP, DBP, cholesterol, triglyceride, SCR, LDL were undeniably related to incident diabetes. Besides, the outcome also 
manifested that men had a higher risk of incident diabetes than women, the participants with family history of diabetes have a higher risk of incident diabetes than without family history, and the participants with current drinking or smoking status have a higher risk of incident diabetes than the participants with never or ever drinking or smoking status. 
Table 3

The results of univariate analysis

\begin{tabular}{|c|c|c|}
\hline & Statistics & HR(95\%Cl,P-value) \\
\hline Age (years) & $42.229 \pm 12.819$ & $1.070(1.066,1.073)<0.00001$ \\
\hline $\mathrm{BMI}\left(\mathrm{kg} / \mathrm{m}^{2}\right)$ & $23.220 \pm 3.303$ & $1.232(1.219,1.245)<0.00001$ \\
\hline FPG (mmol/L) & $4.931 \pm 0.615$ & $10.323(9.655,11.036)<0.00001$ \\
\hline SBP (mmHg) & $119.241 \pm 16.520$ & $1.041(1.039,1.043)<0.00001$ \\
\hline DBP (mmHg) & $74.104 \pm 10.847$ & $1.048(1.044,1.051)<0.00001$ \\
\hline Cholesterol (mmol/L) & $4.693 \pm 0.896$ & $1.431(1.370,1.495)<0.00001$ \\
\hline Triglyceride (mmol/L) & $1.319 \pm 1.011$ & $1.258(1.242,1.275)<0.00001$ \\
\hline SCR (umol/L) & $71.207 \pm 15.691$ & $1.005(1.004,1.007)<0.00001$ \\
\hline ALT/AST & $0.917 \pm 0.358$ & $3.176(2.847,3.542)<0.00001$ \\
\hline \multicolumn{3}{|l|}{ Gender } \\
\hline male & $48942(56.477 \%)$ & 1.0 \\
\hline female & $37717(43.523 \%)$ & $0.574(0.519,0.634)<0.00001$ \\
\hline \multicolumn{3}{|l|}{$\mathrm{HDL}(\mathrm{mmol} / \mathrm{L})$} \\
\hline $\operatorname{low}(0$ to $\leq 1.23)$ & $16272(18.777 \%)$ & 1.0 \\
\hline middle(1.24 to $\leq 1.46)$ & $15822(18.258 \%)$ & $0.997(0.871,1.142) 0.96748$ \\
\hline $\operatorname{high}(1.47$ to $\leq 10.40)$ & $16965(19.577 \%)$ & $0.933(0.816,1.066) 0.30476$ \\
\hline not recorded & $37600(43.388 \%)$ & $0.531(0.471,0.599)<0.00001$ \\
\hline \multicolumn{3}{|l|}{ LDL (mmol/L) } \\
\hline $\operatorname{low}(0$ to $\leq 2.42)$ & $16381(18.903 \%)$ & 1.0 \\
\hline middle(2.42 to $\leq 2.98)$ & $16759(19.339 \%)$ & $1.138(0.985,1.314) 0.07886$ \\
\hline high(2.99 to $\leq 10.07)$ & $16758(19.338 \%)$ & $1.572(1.373,1.799)<0.00001$ \\
\hline not recorded & $36761(42.420 \%)$ & $0.628(0.549,0.719)<0.00001$ \\
\hline \multicolumn{3}{|c|}{ Family history of diabetes } \\
\hline no & $84915(97.988 \%)$ & 1.0 \\
\hline yes & $1744(2.012 \%)$ & $1.632(1.279,2.084) 0.00008$ \\
\hline
\end{tabular}

Values are $n(\%)$, median(Q1-Q3) or mean \pm sd 


\begin{tabular}{|lll|}
\hline & Statistics & HR(95\%Cl,P-value $)$ \\
\hline Smoking status & & \\
\hline current & $4085(4.714 \%)$ & 1.0 \\
\hline ever & $965(1.114 \%)$ & $0.909(0.612,1.352) 0.63882$ \\
\hline never & $16642(19.204 \%)$ & $0.510(0.413,0.629)<0.00001$ \\
\hline not recorded & $64967(74.969 \%)$ & $0.650(0.544,0.777)<0.00001$ \\
\hline Drinking status & & \\
\hline current & $571(0.659 \%)$ & 1.0 \\
\hline ever & $3747(4.324 \%)$ & $0.443(0.276,0.710) 0.00072$ \\
\hline never & $17374(20.049 \%)$ & $0.407(0.264,0.628) 0.00005$ \\
\hline not recorded & $64967(74.969 \%)$ & $0.443(0.291,0.675) 0.00015$ \\
\hline Values are $n(\%)$, median(Q1-Q3) or mean \pm sd & \\
\hline
\end{tabular}

The Kaplan-Meier curves of the cumulative hazards of incident diabetes risk was depicted in Fig. 1 when stratified by quartiles of ALT/AST. Incident diabetes risk between each quartile of ALT/AST was evidentially various (log-rank test, $p<0.0001$ ). When ALT/AST increased, the cumulative incident diabetes risk raised by degrees, and the Q4 ALT/AST (top of the quartiles) was notarized having maximum risk of incident diabetes.

\section{Association of ALT/AST and incident diabetes}

The association of ALT/AST and incident diabetes was judged by Cox proportional hazard regression model in which Table 4 demonstrated the non-adjusted and adjusted models. ALT/AST was observed positively associate with incident diabetes ( $\mathrm{HR}=3.176,95 \%$ confidence interval $(\mathrm{Cl}): 2.847$ to $3.542, \mathrm{P}<$ 0.00001 ) in crude model. Similar outcome was not only demonstrated in minimally adjusted model (adjusted age, gender, BMI, DBP, SBP, family history of diabetes, smoking status, drinking status) with (HR:3.434, 95\% Cl:2.980 to 3.958, $\mathrm{P}<0.00001$ ), but also in full adjusted model (adjusted age, gender, BMI, DBP, SBP, family history of diabetes, smoking status, drinking status, FPG, HDL, LDL, cholesterol, triglyceride, SCR) with ( $\mathrm{HR}=2.535,95 \% \mathrm{Cl}: 2.190$ to $2.934, \mathrm{P}<0.00001)$. In addition, when ALT/AST was conducted as a categorical variable by quartiles, the Q4 was detected 1.04 times ascendant of the risk of incident diabetes than Q1 in full adjusted model with (P for trend $=0.00007)$. 
Table 4

Relationship between AST/ALT and censor of diabetes at follow up in different models

\begin{tabular}{|c|c|c|c|}
\hline Exposure & $\begin{array}{l}\text { Crude Model (HR, } \\
95 \% \mathrm{Cl}, \mathrm{P})\end{array}$ & $\begin{array}{l}\text { Minimally adjusted Model } \\
(\mathrm{HR}, 95 \% \mathrm{Cl}, \mathrm{P})\end{array}$ & $\begin{array}{l}\text { Fully adjusted Model } \\
\text { (HR, } 95 \% \mathrm{Cl}, \mathrm{P})\end{array}$ \\
\hline $\begin{array}{l}\text { ALT/AST (per0.1 } \\
\text { charge) }\end{array}$ & $\begin{array}{l}3.176(2.847,3.542) \\
<0.00001\end{array}$ & $\begin{array}{l}3.434(2.980,3.958)< \\
0.00001\end{array}$ & $\begin{array}{l}2.535(2.190,2.934)< \\
0.00001\end{array}$ \\
\hline \multicolumn{4}{|l|}{$\begin{array}{l}\text { ALT/AST } \\
\text { (quartile) }\end{array}$} \\
\hline Q1 & 1.0 & 1.0 & 1.0 \\
\hline Q2 & $\begin{array}{l}1.373(1.152,1.636) \\
0.00040\end{array}$ & $\begin{array}{l}1.207(1.011,1.441) \\
0.03726\end{array}$ & $\begin{array}{l}1.031(0.863,1.231) \\
0.73672\end{array}$ \\
\hline Q3 & $\begin{array}{l}2.325(1.978,2.734) \\
<0.00001\end{array}$ & $\begin{array}{l}1.851(1.565,2.191)< \\
0.00001\end{array}$ & $\begin{array}{l}1.403(1.185,1.661) \\
0.00009\end{array}$ \\
\hline Q4 & $\begin{array}{l}3.521(3.019,4.107) \\
<0.00001\end{array}$ & $\begin{array}{l}3.037(2.553,3.612)< \\
0.00001\end{array}$ & $\begin{array}{l}2.042(1.717,2.429)< \\
0.00001\end{array}$ \\
\hline$P$ for Trend & $<0.00001$ & $<0.00001$ & 0.00007 \\
\hline \multicolumn{4}{|c|}{ Crude model: we did not adjust other covariant } \\
\hline \multicolumn{4}{|c|}{$\begin{array}{l}\text { Minimally adjusted model: we adjusted age, gender, BMI, DBP, SBP, family history of diabetes, } \\
\text { smoking status, drinking status }\end{array}$} \\
\hline \multicolumn{4}{|c|}{$\begin{array}{l}\text { Fully adjusted model: we adjusted age, gender, BMI, DBP, SBP, family history of diabetes, smoking } \\
\text { status, drinking status, FPG, HDL, LDL, cholesterol, triglyceride, SCR }\end{array}$} \\
\hline \multicolumn{4}{|c|}{ Cl: confidence, Ref: reference } \\
\hline
\end{tabular}

\section{Analysis of generalized additive models (GAM)}

As ALT/AST ratio was a continuous variable, the non-linear relationship of ALT/AST and incident diabetes was identified through generalized additive models (GAM). In Table 5 and Fig. 2, we figured out the inflection point of ALT/AST was 0.68 (Log-likelihood ratio test $P=0.176$ ) through a two-piecewise linear regression model (after adjusting age, gender, BMI, FPG, SBP, DBP, cholesterol, triglyceride, SCR, $\mathrm{HDL}$, LDL, family history of diabetes, smoking and drinking statuses). A undeniable relationship between ALT/AST and incident diabetes was detected on both the left side (HR:1.317, 95\%Cl: 0.514-3.375, P = 0.5665 ) and right side (HR: $2.641,95 \% \mathrm{Cl}: 2.257-3.091, \mathrm{P}<0.0001)$ of the inflection point. As for the $P$ value for Log-likelihood ratio test is above 0.05 , so the relation between ALT/AST and incident diabetes is only linear. That means we used generalized linear models (Cox proportional hazard models) to analyze the relation between ALT/AST and incident diabetes is appropriate. 
Table 5

The results of two-piecewise linear regression model incident diabetes $(\mathrm{HR}, 95 \% \mathrm{Cl}, \mathrm{P})$

Fitting model by standard linear regression $2.535(2.190,2.934)<0.0001$

Fitting model by two-piecewise linear regression

Inflection point 0.68

$\leq 0.68$ $1.317(0.514,3.375) 0.5665$

$\bowtie 0.68$ $2.641(2.257,3.091)<0.0001$

P for log likelihood ratio test 0.176

$\mathrm{Cl}$ : Confidence interval

We adjusted age, gender, BMI, DBP, SBP, family history of diabetes, smoking status, drinking status, FPG, HDL, LDL, cholesterol,triglyceride, CCR

\section{Subgroup analysis}

In Table 6, subgroup analysis was used to examined if other variables (age, gender, BMI, FPG, SBP, DBP, HDL, LDL, family history of diabetes, smoking status, drinking status) would affect the relation between ALT/AST and incident diabetes. By treating them as stratified variables, we could study their impact on the association of ALT/AST and incident diabetes. As the output result depicted, age, BMI and SBP were detected interactional through the priori specification test (all $P$ values for interaction $<0.05$ ). The population with age (30 to $<40$ years), BMI $(<24 \mathrm{~kg} / \mathrm{m} 2)$ and SBP $(<140 \mathrm{mmHg}$ ) was found to be the stronger association. On the contrary, the population with age ( 20 to $<30$ years, $>50$ years), BMI ( $\geq$ $24 \mathrm{~kg} / \mathrm{m}^{2}$ ) and SBP ( $\geq 140 \mathrm{mmHg}$ ) was found to be the weaker association. 
Table 6

Effect size of ALT/AST on incident diabetes in prespecified and exploratory subgroups

\begin{tabular}{|c|c|c|}
\hline Characteristic & No of participants & $\mathrm{HR}(95 \% \mathrm{Cl}) \mathrm{P}$ value $\mathrm{P}$ for interacion \\
\hline Age(years) & 11723 & 0.0384 \\
\hline 20 to $<30$ & 33801 & $1.802(0.425,7.578) 0.4218$ \\
\hline 30 to $<40$ & 18235 & $3.502(2.354,5.211) \rrbracket 0.0001$ \\
\hline 40 to $<50$ & 12273 & $2.597(1.906,3.537) \bowtie 0.0001$ \\
\hline 50 to $<60$ & 7668 & $1.985(1.533,2.570) \bowtie 0.0001$ \\
\hline 60 to $<70$ & 2959 & $1.583(1.115,2.248) 0.0101$ \\
\hline$\geq 70$ & & $1.567(0.866,2.835) 0.1375$ \\
\hline Gender & & 0.0999 \\
\hline male & 48942 & $2.394(2.038,2.814)<0.0001$ \\
\hline female & 37717 & $3.091(2.348,4.069)<0.0001$ \\
\hline BMI $\left(\mathrm{kg} / \mathrm{m}^{2}\right)$ & & 0.0013 \\
\hline$<18.5$ & 4894 & $5.365(0.611,47.123) 0.1297$ \\
\hline$\geq 18.5,<24$ & 47961 & $3.726(2.774,5.006)<0.0001$ \\
\hline$\geq 24,<28$ & 26663 & $2.521(2.049,3.100)<0.0001$ \\
\hline$\geq 28$ & 7141 & $1.649(1.247,2.181) 0.0004$ \\
\hline FPG (mmol/L) & & 0.0880 \\
\hline $\operatorname{low}(1.78$ to $\leq 4.92)$ & 43228 & $2.171(1.413,3.335) 0.0004$ \\
\hline high(4.93 to $\leq 6.99)$ & 43431 & $3.182(2.738,3.698)<0.0001$ \\
\hline $\mathrm{SBP}(\mathrm{mmHg})$ & & 0.0003 \\
\hline$<140$ & 77553 & $2.944(2.485,3.487)<0.0001$ \\
\hline$\geq 140$ & 9106 & $1.660(1.266,2.178) 0.0002$ \\
\hline $\mathrm{DBP}(\mathrm{mmHg})$ & & 0.1968 \\
\hline$<90$ & 79689 & $2.667(2.265,3.141)<0.0001$ \\
\hline$\geq 90$ & 6970 & $2.127(1.564,2.893)<0.0001$ \\
\hline $\mathrm{HDL}$ (mmol/L) & & 0.4873 \\
\hline
\end{tabular}

Note 1: Above model adjusted for age, gender, BMI, FPG, SBP, DBP, HDL, LDL, family history of diabetes, cholesterol, triglyceride, SCR, smoking status, drinking status 


\begin{tabular}{|c|c|c|}
\hline Characteristic & No of participants & $\mathrm{HR}(95 \% \mathrm{Cl}) \mathrm{P}$ value $\mathrm{P}$ for interacion \\
\hline $\operatorname{low}(0$ to $\leq 1.23)$ & 16272 & $2.957(2.308,3.787)<0.0001$ \\
\hline middle(1.24 to $\leq 1.46)$ & 15822 & $2.462(1.839,3.295)<0.0001$ \\
\hline $\operatorname{high}(1.47$ to $\leq 10.40)$ & 16965 & $2.491(1.824,3.402)<0.0001$ \\
\hline not recorded & 37600 & $2.310(1.855,2.877)<0.0001$ \\
\hline LDL (mmol/L) & & 0.2055 \\
\hline $\operatorname{low}(0$ to $\leq 2.42)$ & 16381 & $3.285(2.368,4.556)<0.0001$ \\
\hline middle(2.42 to $\leq 2.98)$ & 16759 & $2.801(2.042,3.841)<0.0001$ \\
\hline high(2.99 to $\leq 10.07)$ & 16758 & $2.184(1.668,2.861)<0.0001$ \\
\hline not recorded & 36761 & $2.314(1.811,2.956)<0.0001$ \\
\hline Family history of diabetes & & 0.7528 \\
\hline no & 84915 & $2.542(2.189,2.951)<0.0001$ \\
\hline yes & 1744 & $2.254(1.082,4.697) 0.0300$ \\
\hline Smoking status & & 0.1793 \\
\hline current & 4085 & $1.507(0.918,2.475) 0.1052$ \\
\hline ever & 965 & $2.425(0.817,7.197) 0.1103$ \\
\hline never & 16642 & $2.420(1.664,3.521)<0.0001$ \\
\hline not recorded & 64967 & $2.686(2.272,3.176)<0.0001$ \\
\hline Drinking status & & 0.8861 \\
\hline current & 571 & $1.632(0.356,7.474) 0.5281$ \\
\hline ever & 3747 & $2.406(1.284,4.510) 0.0062$ \\
\hline never & 17374 & $2.391(1.693,3.375)<0.0001$ \\
\hline not recorded & 64967 & $2.633(2.225,3.117)<0.0001$ \\
\hline
\end{tabular}

\section{Discussion}

In the present study, by using the generalized linear models (Cox proportional hazard models), ALT/AST was observed positively associate with incident diabetes when other variables were adjusted. Moreover, the analyses of generalized additive models (GAM) demonstrated that the relation between ALT/AST and 
incident diabetes is only linear. Subgroup analysis was conducted to further disclosure the association of ALT/AST and incident diabetes in hierarchical populations. The population with age (30 to < 40 years), BMI $(<24 \mathrm{~kg} / \mathrm{m} 2)$ and SBP $(<140 \mathrm{mmHg})$ was found to be the stronger association. On the contrary, the population with age ( 20 to $<30$ years, $>50$ years), $\mathrm{BMI}\left(\geq 24 \mathrm{~kg} / \mathrm{m}^{2}\right)$ and SBP $(\geq 140 \mathrm{mmHg}$ ) was found to be the weaker association.

In former study, ALT/AST has been brought into evaluate insulin resistance[7, 14, 15]. Hanley et al. have informed that ALT/AST could be used for assessing metabolic syndrome through decide insulin sensitivity and acute insulin response $[16,17]$. Besides, former researches have confirmed that hepatic diseases like non-alcoholic fatty liver disease (NAFLD) were related to glucose metabolism and the prevalence of diabetes[18, 19]. And the elevation of liver enzymes such as ALT, AST and gamma-glutamyl transpeptidase (GGT) or the ALT/AST ratio are treated as surrogate markers of NAFLD in common [2022]. Monami, M., et al. have affirmed a undeniable relation between elevated liver enzyme levels and type 2 diabetes (T2DM) [23]. Wen JP, et al. have revealed that the increasement of serum GGT quartiles were positively related to the prevalence of T2DM after adjustment for prospective confounders in Chinese population [24]. Former research showed that ALT could be used for assessing the risk of incident diabetes independent of the fasting glucose, adiposity and family history of diabetes [25]. And in a prospective cohort study which performed an analyses of 6484 participants with age ( $\geq 40$ years old) in Korean indicated that individuals with high aminotransferase levels, the risk of incident diabetes was three times higher in those who have highest quartile of BMI than those in the lowest quartile of BMI[26]. Yet, only a few investigations have focused considerably the association between ALT/AST and incident diabetes in the Chinese population. Thus, our study had huge objects $(685,277)$ across 32 sites and 11 cities in China that could represent Chinese population rigorously. And the results creatively suggested that ALT/AST is an independent risk factor for incident diabetes in Chinese adults.

Subgroup analysis is extraordinary important for a retrospective cohort analysis. Subgroup analysis was generally used for investigating the coherence of the clinical trial's result among various subpopulations defined by each of multiple baseline characteristics of the patients or assessing the treatment effect of special characteristics of patients that usually was treated as the chiefly or second research aim[27, 28]. In the present study, we used age, gender, BMI, FPG, SBP, DBP, HDL, LDL, family history of diabetes, smoking status, drinking status as the stratified variables, of which only age, BMI and SBP were found that they could strengthen or weaken the association between ALT/AST and incident diabetes. The possible reason for the above results is worth further exploration. Maybe the age, BMI or SBP is the intermediate factor between ALT/AST and incident diabetes in Chinese adults that they modified the effect of ALT/AST ratio on incident diabetes or the different levels of themselves could have imparity effect on ALT/AST ratio or incident diabetes. For example, a study which enrolled 1732 young adults with $(n=287)$ and without $(n=1445)$ insulin resistance indicated that insulin resistance is related to elevated transaminases and low AST/ALT ratio in young adults with normal weight[29]. The clinical value of this study is as follows: (1) As far as we know, it is the first time to reveal the association of ALT/AST ratio 
and incident diabetes in Chinese adults; (2) The results of the present article would contribute to further study on the structure of diagnostic or predictive models of incident diabetes.

There were several strengths in the present study. In the first place, compared with previous similar studies, the sample size of our study is relatively bigger. What's more, we assessed the linearity during research. Besides, there would be prone to confusion as the nature of observational study. For this reason, rigorous adjusting of the component confounders was conducted for reducing confusion. Moreover, in order to decrease the natural event during analysis and strengthen the characteristic of outcome, ALT/AST was conducted as not only continuous variable but also categorical variable. Last but not least, the analysis of the modification factors could make sure the results more typical in various subgroups.

Nonetheless, some limitations deserved to be noted. To begin with, the data we analyzed have already been examined by Chen et al, our study was a second retrospective cohort study which was conducted by the Rich Healthcare Group[10]. Owing to the confined raw data, our findings might be not generalizable to other groups. In the meantime, the present research was a second retrospective cohort study based on published data, so the variable which not consisted in the data set was unable to be adjusted.

Furthermore, as ALT/AST was measured at baseline, the changes during following up are not obsessed in the present study. Additionally, even if an extensive set of confounding factors have been adjusted, but the influence of the residual confounding factors which because of the measuring errors in the categorization or the unmeasured factors such as dietary habits or physical exercise can't be obviated. Consequently, a further investigation in a longer following up with more fastidious regulations is required.

\section{Declarations}

Funding: This study was funded by National Natural Science Foundation of China (Grant No: 31770984, 81929001), Shenzhen Science and Technology Peacock Team Project (Grant No: KQTD2017 0331145453160), Innovation of Science and Technology Commission of Shenzhen Municipality (Grant No: JCYJ20170412170814624), Shenzhen Nanshan District Pioneer Group Research Funds (Grant No: LHTD20180007).

Conflicts of interest: Not applicable.

Ethics approval: On account of the character of retrospective cohort analysis, informed consent or study approval of the patient was not demanded by the institutional ethics committee.

Consent to participate: Not applicable.

Consent for publication: Not applicable.

Availability of data and material: All data generated or analysed during this study are included in this published article. 
Code availability: Not applicable.

Authors' contributions: Zhilin Liang, Haofei Hu and Xunxun Wang made substantial contributions to the conception and design of the work, the analysis and interpretation of data. Zhilin Liang drafted the work or revised it critically for important intellectual content. Xianxiong Chen approved the version to be published. Min Zhang and Zhigang Liu have contributed to the funding acquisition and project administration. Zhilin Liang and Zhigang Liu agree to be accountable for all aspects of the work in ensuring that questions related to the accuracy or integrity of any part of the work are appropriately investigated and resolved.

\section{References}

1. Wang L, Gao P, Zhang M, Huang Z, Zhang D, Deng Q, et al. Prevalence and Ethnic Pattern of Diabetes and Prediabetes in China in 2013. Jama. 2017;317(24):2515-23. doi:10.1001/jama.2017.7596.

2. Ogurtsova K, da Rocha Fernandes JD, Huang Y, Linnenkamp U, Guariguata L, Cho NH, et al. IDF Diabetes Atlas: Global estimates for the prevalence of diabetes for 2015 and 2040. Diabetes Res Clin Pract. 2017;128:40-50. doi:10.1016/j.diabres.2017.03.024.

3. Ko SH, Baeg MK, Han KD, Ko SH, Ahn YB. Increased liver markers are associated with higher risk of type 2 diabetes. World J Gastroenterol. 2015;21(24):7478-87. doi:10.3748/wjg.v21.i24.7478.

4. Felig P. The glucose-alanine cycle. Metabolism. 1973;22(2):179-207. doi:10.1016/00260495(73)90269-2.

5. Sheng X, Che H, Ji Q, Yang F, Lv J, Wang Y, et al. The Relationship Between Liver Enzymes and Insulin Resistance in Type 2 Diabetes Patients with Nonalcoholic Fatty Liver Disease. Horm Metab Res. 2018;50(5):397-402. doi:10.1055/a-0603-7899.

6. Fraser A, Harris R, Sattar N, Ebrahim S, Davey Smith G, Lawlor DA. Alanine aminotransferase, gamma-glutamyltransferase, and incident diabetes: the British Women's Heart and Health Study and meta-analysis. Diabetes Care. 2009;32(4):741-50. doi:10.2337/dc08-1870.

7. Kawamoto R, Kohara K, Kusunoki T, Tabara Y, Abe M, Miki T. Alanine aminotransferase/aspartate aminotransferase ratio is the best surrogate marker for insulin resistance in non-obese Japanese adults. Cardiovasc Diabetol. 2012;11:117. doi:10.1186/1475-2840-11-117.

8. Vozarova B, Stefan N, Lindsay RS, Saremi A, Pratley RE, Bogardus C, et al. High alanine aminotransferase is associated with decreased hepatic insulin sensitivity and predicts the development of type 2 diabetes. Diabetes. 2002;51(6):1889-95. doi:10.2337/diabetes.51.6.1889.

9. Hanley AJ, Williams K, Festa A, Wagenknecht LE, D'Agostino RB Jr, Kempf J, et al. Elevations in markers of liver injury and risk of type 2 diabetes: the insulin resistance atherosclerosis study. Diabetes. 2004;53(10):2623-32. doi:10.2337/diabetes.53.10.2623.

10. Chen Y, Zhang XP, Yuan J, Cai B, Wang XL, Wu XL, et al. Association of body mass index and age with incident diabetes in Chinese adults: a population-based cohort study. BMJ open. 2018;8(9):e021768. doi:10.1136/bmjopen-2018-021768. 
11. Erviti J, Alonso A, Oliva B, Gorricho J, Lopez A, Timoner J, et al. Oral bisphosphonates are associated with increased risk of subtrochanteric and diaphyseal fractures in elderly women: a nested casecontrol study. BMJ Open. 2013;3(1). doi:10.1136/bmjopen-2012-002091.

12. von Elm E, Altman DG, Egger M, Pocock SJ, Gotzsche PC, Vandenbroucke JP. The Strengthening the Reporting of Observational Studies in Epidemiology (STROBE) statement: guidelines for reporting observational studies. Lancet. 2007;370(9596):1453-7. doi:10.1016/s0140-6736(07)61602-x.

13. Vandenbroucke JP, von Elm E, Altman DG, Gotzsche PC, Mulrow CD, Pocock SJ, et al. Strengthening the Reporting of Observational Studies in Epidemiology (STROBE): explanation and elaboration. Int J Surg. 2014;12(12):1500-24. doi:10.1016/j.ijsu.2014.07.014.

14. Zhao L, Cheng J, Chen Y, Li Q, Han B, Chen Y, et al. Serum alanine aminotransferase/aspartate aminotransferase ratio is one of the best markers of insulin resistance in the Chinese population. Nutr Metab (Lond). 2017;14:64. doi:10.1186/s12986-017-0219-x.

15. Kwon SS, Lee SG. A High Alanine Aminotransferase/Aspartate Aminotransferase Ratio Determines Insulin Resistance and Metabolically Healthy/Unhealthy Obesity in a General Adult Population in Korea: The Korean National Health and Nutritional Examination Survey 2007-2010. Exp Clin Endocrinol Diabetes. 2019;127(10):677-84. doi:10.1055/a-0752-0217.

16. Hanley AJ, Wagenknecht LE, Festa A, D'Agostino RB Jr, Haffner SM. Alanine aminotransferase and directly measured insulin sensitivity in a multiethnic cohort: the Insulin Resistance Atherosclerosis Study. Diabetes Care. 2007;30(7):1819-27. doi:10.2337/dc07-0086.

17. Homsanit M, Sanguankeo A, Upala S, Udol K. Abnormal liver enzymes in Thai patients with metabolic syndromes. J Med Assoc Thai. 2012;95(3):444-51.

18. Andre P, Balkau B, Born C, Royer B, Wilpart E, Charles MA, et al. Hepatic markers and development of type 2 diabetes in middle aged men and women: a three-year follow-up study. The D.E.S.I.R. Study (Data from an Epidemiological Study on the Insulin Resistance syndrome). Diabetes Metab. 2005;31(6):542-50. doi:10.1016/s1262-3636(07)70229-x.

19. Baig NA, Herrine SK, Rubin R. Liver disease and diabetes mellitus. Clin Lab Med. 2001;21(1):193207.

20. Ballestri S, Zona S, Targher G, Romagnoli D, Baldelli E, Nascimbeni F, et al. Nonalcoholic fatty liver disease is associated with an almost twofold increased risk of incident type 2 diabetes and metabolic syndrome. Evidence from a systematic review and meta-analysis. J Gastroenterol Hepatol. 2016;31(5):936-44. doi:10.1111/jgh.13264.

21. Lee $\mathrm{K}$, Yang JH. Which liver enzymes are better indicators of metabolic syndrome in adolescents: the Fifth Korea National Health and Nutrition Examination Survey, 2010. Metab Syndr Relat Disord. 2013;11(4):229-35. doi:10.1089/met.2012.0153.

22. Martin-Rodriguez JL, Gonzalez-Cantero J, Gonzalez-Cantero A, Arrebola JP, Gonzalez-Calvin JL. Diagnostic accuracy of serum alanine aminotransferase as biomarker for nonalcoholic fatty liver disease and insulin resistance in healthy subjects, using 3T MR spectroscopy. Medicine. 2017;96(17):e6770. doi:10.1097/md.0000000000006770. 
23. Monami M, Bardini G, Lamanna C, Pala L, Cresci B, Francesconi P, et al. Liver enzymes and risk of diabetes and cardiovascular disease: results of the Firenze Bagno a Ripoli (FIBAR) study. Metabolism. 2008;57(3):387-92. doi:10.1016/j.metabol.2007.10.015.

24. Wen J, Liang Y, Wang F, Sun L, Guo Y, Duan X, et al. C-reactive protein, gamma-glutamyltransferase and type 2 diabetes in a Chinese population. Clin Chim Acta. 2010;411(3-4):198-203. doi:10.1016/j.cca.2009.11.002.

25. Lorenzo C, Hanley AJ, Rewers MJ, Haffner SM. Discriminatory value of alanine aminotransferase for diabetes prediction: the Insulin Resistance Atherosclerosis Study. Diabet Med. 2016;33(3):348-55. doi:10.1111/dme.12835.

26. Song BM, Kim HC, Kim DJ, Ahn SV, Kim KM, Lee JM, et al. Aminotransferase levels, body mass index, and the risk of diabetes: a prospective cohort study. Ann Epidemiol. 2018;28(10):675 - 80.e6. doi:10.1016/j.annepidem.2018.07.009.

27. Sacks FM, Pfeffer MA, Moye LA, Rouleau JL, Rutherford JD, Cole TG, et al. The effect of pravastatin on coronary events after myocardial infarction in patients with average cholesterol levels. Cholesterol and Recurrent Events Trial investigators. N Engl J Med. 1996;335(14):1001-9. doi:10.1056/nejm199610033351401.

28. Jackson RD, LaCroix AZ, Gass M, Wallace RB, Robbins J, Lewis CE, et al. Calcium plus vitamin D supplementation and the risk of fractures. N Engl J Med. 2006;354(7):669-83. doi:10.1056/NEJMoa055218.

29. Simental-Mendia LE, Rodriguez-Moran M, Gomez-Diaz R, Wacher NH, Rodriguez-Hernandez H, Guerrero-Romero F. Insulin resistance is associated with elevated transaminases and low aspartate aminotransferase/alanine aminotransferase ratio in young adults with normal weight. Eur $\mathrm{J}$ Gastroenterol Hepatol. 2017;29(4):435-40. doi:10.1097/meg.0000000000000811. 